\title{
Beatrice E. KITZINGER, The Cross, the Gospels, and the Work of Art in the Carolingian Age
}

Éric Palazzo

\section{(2) OpenEdition}

\section{Journals}

Édition électronique

URL : https://journals.openedition.org/ccm/5087

DOI : $10.4000 / \mathrm{ccm} .5087$

ISSN : 2119-1026

\section{Éditeur}

Centre d'études supérieures de civilisation médiévale/Université de Poitiers

\section{Édition imprimée}

Date de publication : 1 septembre 2020

Pagination : 178-180

ISBN : 978-2-490783-06-9

ISSN : 0007-9731

Référence électronique

Éric Palazzo, « Beatrice E. kItzInger, The Cross, the Gospels, and the Work of Art in the Carolingian Age », Cahiers de civilisation médiévale [En ligne], 250-251 | 2020, mis en ligne le 01 septembre 2020, consulté le 02 décembre 2022. URL : http://journals.openedition.org/ccm/5087 ; DOI : https://doi.org/10.4000/ ccm.5087

\section{(c) (i) (9)}

Creative Commons - Attribution - Pas d'Utilisation Commerciale - Pas de Modification 4.0 International - CC BY-NC-ND 4.0

https://creativecommons.org/licenses/by-nc-nd/4.0/ 
Beatrice E. Kitzinger, The Cross, the Gospels, and the Work of Art in the Carolingian Age, Cambridge, Cambridge Universtiy Press, 2019.

Il est rare de pouvoir affirmer qu'un ouvrage sur l'image médiévale et, de façon plus large, sur la culture visuelle du Moyen Âge, s'imposera rapidement comme un « classique » tant l'ampleur de l'enquête, la qualité du travail produit comme la nouveauté des résultats sautent aux yeux du lecteur après avoir refermé le livre dont la qualité matérielle contribue, elle aussi, à son appréciation très positive. À la lecture du titre de l'ouvrage, on pourrait au premier abord penser que le sujet n'est pas neuf, ayant pour effet d'aiguiser la curiosité pour l'enquête menée par Beatrice Kitzinger. Nombre de publications ont depuis plusieurs décennies largement traité de la croix dans l'art du haut Moyen Âge, ou bien de l'illustration des livres d'évangiles à l'époque carolingienne et au-delà au X ${ }^{\mathrm{e}} \mathrm{s}$. Autrement dit, B. Kitzinger s'est attaquée à un matériau a priori bien connu des spécialistes de l'art du haut Moyen Âge et, de façon plus générale, des médiévistes.

La première grande originalité du livre est de proposer une approche renouvelée de ce matériau à partir d'une compréhension théorique qui s'appuie sur une connaissance sans faille de la théologie et de la liturgie du haut Moyen Âge. Non pas que par le passé, les auteurs - du moins pour certains - n'aient pas tenu compte du contexte théologique et liturgique de la croix et des livres d'évangiles et leurs illustrations dans le haut Moyen Âge, mais B. Kitzinger propose une cadre théologico-liturgique neuf et inédit pour comprendre ce matériau qui se révèle non seulement très convaincant mais aussi très suggestif pour repenser, dans l'avenir, certaines œuvres de l'art médiéval au-delà de la période carolingienne. Avant d'entrer dans le détail de la démonstration, 
quelques observations d'ordre général sur la méthode et l'historiographie du sujet.

À propos de l'historiographie des recherches sur la croix et les livres d'évangiles du haut Moyen Âge, le lecteur a bien du mal à trouver des lacunes tant l'a. s'appuie sur une connaissance plus qu'approfondie de la bibliographie et de ses différents apports à la compréhension des thèmes traités. L'a. connaît pour ainsi dire à la perfection les travaux réalisés par d'autres avant elle - et ils sont souvent très nombreux sur de tels sujets - , comme il apparaît clairement dans les notes de chaque chapitre qui sont non seulement l'occasion d'exposer au lecteur le matériau historiographique utilisé mais donnent également l'opportunité pour d'intéressantes digressions d'ordre méthodologique, épistémologique ou théorique en lien direct ou pas avec le sujet du livre ou l'exposé sur tel ou tel objet ou une image particulière. Á ce sujet, il faut savoir gré à l'a. de renouer avec une tradition quelque peu tombée en désuétude, ou même à l'abandon depuis de nombreuses années, et consistant à « produire » du débat intellectuel et savant par des prises de position argumentées et contradictoires avec d'autres auteurs. Autre point fort du livre que l'on doit relever d'emblée : l'attachement de $\mathrm{B}$. Kiztinger à la théologie médiévale et à ses concepts propres. Rien d'étonnant à cela? Pas tant que cela si l'on considère que nombre d'auteurs ont dans un passé récent commis l'erreur d'appréhender la théologie médiévale, et même la liturgie, à partir de concepts forgés par les théoriciens de l'esthétique contemporaine ou par la philosophie moderne. À l'inverse de cela, B. Kitzinger reste au plus près des auteurs médiévaux et de leur pensée, quitte à, souvent avec raison, ne pas traduire des mots latins, comme « utilitas » qu'une traduction réduirait presque à néant la complexité de la notion et que l'a. s'attache avec succès à cerner. À de rares occasions cependant, B. Kitzinger se laisse aller à des formules ou à des expressions « modernes » dont elle aurait pu à mon sens se passer, comme celle de « self reflexivity ». Mais c'est un détail tant le respect que l'a. porte aux textes médiévaux et à leur complexité force l'admiration.

Ne doutons pas que les qualités dont fait preuve l'a. dans son traitement de l'historiographie, de la bibliographie et du maniement des concepts et des notions sauront influencer d'autres publications dans l'avenir. Relevons également la très bonne connaissance dont fait preuve B. Kitzinger de la bibliographie publiée sur le sujet dans différents pays et dans une grande diversité de langues, démontrant ainsi sa dimension internationale.
Venons-en au thème du livre à proprement parler, à l'enquête menée et aux principaux résultats. Comme je l'ai évoqué précédemment, tant et tant de publications ont été réalisées sur le thème de la croix dans l'art du haut Moyen Âge comme sur les livres d'évangiles principalement carolingiens. L'un des grands mérites du livre de B. Kiztinger est de s'appuyer sur ces travaux antérieurs tout en proposant une approche renouvelée très convaincante. L'idée générale est celle du « signum crucis » comme nœud gordien de la conception temporelle de la chrétienté médiévale, où le passé, le présent et le futur se rencontrent de manière à ne former qu'un seul est même temps concentré et concrétisé dans « l'objet» et/ou « l'image ». Comme l'affirme l'a. dans ses pages conclusives, en perspective chrétienne du haut Moyen Âge, la croix est par excellence, le medium du passé, du présent et du futur. Sans qu'elle le traite de façon très explicite, au final, B. Kitzinger souligne comme personne ne l'avait réellement fait jusque-là, ou tout au moins de façon aussi approfondie et poussée, la portée eschatologique de la croix sous toutes ses formes de représentations et de matérialisations. Depuis les croix processionnelles auxquelles l'a. accorde d'admirables pages, en passant par la croix présente dans le thème iconographique de la crucifixion et le rôle joué par cet épisode dans la relation entre le temps de l'eschatologie et celui de «l'historicité narrative », B. Kiztinger suggère que ce «signum » spécifique est l'instrument principal pour la mise en œuvre d'une « rhétorique visuelle » dont les fondements théologiques renvoient directement à l'Incarnation. À lire B. Kiztinger, on pourrait parfois se demander si elle ne mélange pas les supports, les images, les objets, les différents types de manuscrits dans une sorte de mélange des « genres » qui conduirait à la négation des particularismes et des spécificités propres à chaque objet, à chaque thème iconographique ou même à chaque texte théologique ou liturgique se référant à la croix. Il n'en est rien. Bien au contraire. L'a. montre avec force que la diversité des supports, des objets, des « images » ou bien encore des thèmes théologiques et liturgiques relatifs à la croix ne fait finalement que renforcer l'idée majeure selon laquelle la croix est ce médiateur essentiel pour l'expression vivante de la théologie de l'Incarnation. C'est très principalement dans le riche premier chapitre que l'a. développe ses idées sur ce sujet, passant avec beaucoup de dextérité d'un type d'objets et de matériaux pour proposer une lecture et une compréhension très neuve de ces « multiples croix » carolingiennes. Par ex., on ne s'attendait pas à voir les « croix » du traité de Raban Maur sur la louange de la Sainte Croix dialoguer aussi facilement 
avec les croix processionnelles du haut Moyen Âge et d'autres encore peintes dans différents types de manuscrits. Dans ce chapitre, et, de façon générale dans l'ensemble du livre, il faut louer le grand sens de l'érudition de l'a. qui connaît très manifestement les manuscrits et les objets du Moyen Âge. Sur ce point, l'a. démontre sa grande capacité pour une approche pleinement interdisciplinaire des « images médiévales » comme, également, lorsqu'elle traite à armes égales les inscriptions sur les objets et dans les peintures au même titre que l'iconographie et le discours visuel. Dans ce cadre, son approche fait écho aux publications récentes de Vincent Debiais au sujet de ce qu'il a appelé « la croisée des signes ». Dans ce premier chapitre également, comme encore une fois dans l'ensemble de l'ouvrage, l'a. fait preuve d'une connaissance parfaite et de la prise en compte de ce que l'on pourrait appeler les « monuments majeurs » de l'art du haut Moyen Âge - du moins dans le domaine des manuscrits enluminés comme dans celui des objets liturgiques - mais il faut savoir gré à B. Kitzinger d'avoir aussi inclus dans ses démonstrations des œuvres que certains pourraient à tort qualifier de « secondaires », montrant ainsi que le discours théologique et liturgique sur la croix s'est développé dans des productions issues de centres parfois prestigieux - comme les cours impériales ou royales - mais aussi dans certains centres religieux moins prestigieux. Dans le chapitre premier, l'un des morceaux de bravoure est sans conteste l'étude de la série séquentielle de la croix dans le célèbre sacramentaire de Gellone, réalisé à la fin $\mathrm{du} \mathrm{VIII}^{\mathrm{e}} \mathrm{s}$., et dont B. Kiztinger suggère toute la fécondité pour les inventions iconographiques du IX ${ }^{\mathrm{e}} \mathrm{s}$., en particulier dans les sacramentaires de la production issue de l'entourage de Charles le Chauve. Là, l'a. développe intelligemment la mise en action de la croix dans la liturgie et le caractère « actif » des objets au profit de l'expression de la théologie. Dans ces pages, on voit déjà se profiler le discours sur la relation éminement complexe d'un point de vue théologique et liturgique entre la croix et la crucifixion. À ce sujet, B. Kiztinger innove en insistant surtout sur la perspective « narrative » ou « historique » du thème de la crucifixion venant s'ajouter et enrichir l'ample portée théologique du « signum » par excellence qu'est la croix. Cette démonstration est achevée dans le beau chapitre consacré aux évangiles de la fin du IX ${ }^{\mathrm{e}} \mathrm{s}$. ou du début $\mathrm{du} \mathrm{x}^{\mathrm{e}}$ conservés à la Bibliothèque municipale d'Angers (ms. 24 [20]) et sa double composition des folios $7 \mathrm{v}$ et $8 \mathrm{r}$ contenant respectivement la crucifixion et la déposition de croix.

Le chap. 3 aborde de façon neuve et convaincante la relation entre « l'image-croix » et le livre d'évangiles. Ici, l'a. démontre les liens pour ainsi dire consubstantiels entre la croix et le livre, notamment le livre d'évangiles dont on sait qu'il a été pensé au Moyen Âge à partir du corps du Christ. Dans ce même chapitre, on est séduit par les pages consacrées par l'a. aux croix présentes sur les reliures de manuscrits, notamment des livres d'évangiles et de leur implication du point de vue la perception d'une certaine forme de «monumentalité » de la croix à partir de ces déclinaisons dans la peinture murale mais aussi, par ex., sur ces plats de reliure. Dans ce chapitre, les pages consacrées à la croix dans l'Évangile de Matthieu - notamment à partir de l'intersection des lettres « $\mathrm{L}$ » et «I » du début du texte «Liber generationis... » sont particulièrement riches et convaincantes.

De façon générale le livre de $\mathrm{B}$. Kiztinger est très bien construit, tant dans sa structure générale que dans la progression des idées, excepté peut-être pour le chap. 2 dédié à l'étude d'Otfrid de Wissembourg et à la croix dans les évangiles dont on aurait plutôt vu la place à la fin du chap. 1 .

Pour conclure, je ne peux qu'insister sur les très grandes qualités de ce livre novateur à plusieurs égards et qui fera rapidement date dans les études d'art médiéval mais aussi dans des cercles plus larges de médiévistes. Son auteur y fait preuve d'une très grande intelligence de la matière et d'une capacité à « penser son sujet» laissant présager d'autres publications majeures dans l'avenir.

Éric Palazzo

UMR 7302 - CESCM

Université de Poitiers 